\title{
Dynamic Characteristics of a Variable Damping Isolator with Translating Cam
}

\author{
Lan Wang $\mathbb{D}^{1,2}$ Haijun Xing $\mathbb{D}^{1,2}$ and Yongjun Shen ${ }^{1,2}$ \\ ${ }^{1}$ School of Mechanical Engineering, Shijiazhuang Tiedao University, Shijiazhuang 053400, China \\ ${ }^{2}$ State Key Laboratory of Mechanical Behavior and System Safety of Traffic Engineering Structures, \\ Shijiazhuang Tiedao University, Shijiazhuang 053400, China \\ Correspondence should be addressed to Haijun Xing; xinghaijun310@163.com
}

Received 29 November 2021; Accepted 6 January 2022; Published 3 February 2022

Academic Editor: Yaobing Zhao

Copyright (c) 2022 Lan Wang et al. This is an open access article distributed under the Creative Commons Attribution License, which permits unrestricted use, distribution, and reproduction in any medium, provided the original work is properly cited.

\begin{abstract}
A variable damping isolator based on a translating cam designed especially is proposed in this paper. The nonlinear variable damping isolator is mainly comprised of translating cam with a pair of rollers arranged symmetrically and horizontally and two linear dampers. The damping force of this isolator increases with the increase of its vertical displacement. The dynamic equation is established when the variable damping isolator is applied to the active isolation system under simple harmonic excitation. And the dynamic response of the equation is obtained by Harmonic Balance Method. After that, the numerical simulations are conducted to discuss the effects of the parameters of the isolation system on force transmissibility. The results show that the effects on attenuating the resonance and the force transmissibility in the region of high-frequency ratio are superior to that of the corresponding linear damping vibration isolator when appropriate design parameters (cam profile and damping coefficient of the horizontal linear damper) of the variable damping isolator are selected.
\end{abstract}

\section{Introduction}

Vibration isolators have been widely used in engineering fields. The main indexes affecting the isolation performance of the isolators are natural frequency and damping ratio. In the low-frequency ratio (excitation frequency/natural frequency) range, increasing the damping ratio of the linear damping isolator can greatly reduce the resonance of the system. In the high-frequency ratio range, reducing the damping ratio of the linear damping isolator is beneficial to vibration isolation [1-3]. When the excitation is random or the natural frequency of the system changes, a satisfactory isolation performance cannot be achieved by the linear isolator. Therefore, nonlinear damping isolators have attracted the attention of many scholars because of their variable damping coefficients.

Ravindra et al. $[4,5]$ discussed theoretically the dynamic response and performance of the vibration isolator with both stiffness and damping nonlinearity and achieved the dynamic characteristics of the system with a cubic, hard nonlinear restoring force, and combined Coulomb and viscous damping. Lbrahim [6] reviewed the recent advances of nonlinear damping of viscoelastic materials. Teng et al. [7] proposed three different limit control schemes: large damping TMD system, switching damping limit control, and off-site damping limit control in controlling wind-induced vibration of high-rise buildings. He et al. [8] used twostage variable damping TMD to control the wind-induced vibration of circular high-rise structures and achieved good results. Zhang and Zhuu $[9,10]$ introduced a reusable SMA hysteretic damper whose hysteretic characteristics could be adjusted according to the seismic grade so that it could effectively reduce the vibration amplitude of the building structure under strong earthquakes. Gong and Zhou [11] conducted tests on a three-story steel frame structure with viscoelastic dampers and performed the nonlinear dynamic time-history simulation and analysis. Huang et al. [12-14] contrived a recentering deformation-amplified SMA damper(RDASD), which could amplify the displacement according to the actual engineering requirements to make 
the SMA material work at full energy dissipation capacity. Rabinow [15] discovered the magnetorheological phenomenon first in the 1940s. Subsequently, magnetorheological fluid was used in dampers [16-18]. Wang et al. [19] studied the influence of damping, stiffness, and damping clearance of its hydraulic yaw damper on vehicle stability and wheelrail force. To find out the damping characteristics of semiactive magnetorheological yaw dampers, Mousavi-Bideleh and Berbyuk [20] applied the global sensitivity analysis, multistep approach based on genetic algorithm, and Simpack/Matlab cosimulation to optimize the railway vehicle dynamics performance. Wang et al. [21] studied the performance of ferromagnetic liquid damper under low-frequency vibration. Jiao et al. [22] discussed the nonlinear dynamic characteristics of microvibrating fluid viscous damper considering the entrance effect in the orifice. Jin et al. [23] analyzed the vehicle dynamic performance of high-speed trains with different wheel-rail contact states using variable stiffness and damping semiactive suspension magnetorheological yaw dampers. Yu et al. [24] devised the magnetorheological isolator with variable damping and variable stiffness (MR VDVS) and developed an algebraic parametric model to predict its hysteretic characteristics. Peng et al. [25, 26] found that cubic damping vibration isolator had advantages only in some special cases. Liu et al. [27] stated that the geometric nonlinear friction damping system could significantly reduce the formant of the system while maintaining the attenuation effect on high-frequency vibration and could broaden the adaptive range of the system for the various excitation amplitudes. Vu et al. [28] established a hydraulic damper model of an electronic servo valve for a heavy vehicle and verified the accuracy of the model by the experimental data. Domenico and Ricciardi [29] employed a new equal energy non-Gaussian random linearization method to solve the problem of nonlinear constitutive properties in the study of the seismic performance of energy dissipation structures of nonlinear fluid viscous dampers. Wang et al. [30] optimized the design of the control valve structure of the hydraulic damper according to the optimization objective function of the model and improved the locking response effect of the damper control valve. $\mathrm{Yu}$ et al. [31] found that the shear thickening fluid (STF) isolator exhibited small damping in high vibration frequency and very strong damping at resonance frequency with the increasing of shearing rate. Yan et al. [32] proposed the damping force flow index to determine the damping force of double-out rod hydraulic pore-type viscous damper in the ship-borne equipment. Zhao et al. [33] developed a new type of variable damping TMD which could control the acceleration and displacement response of the structure, and its damping force was gradually enhanced with the increasing of the amplitude of ground motion when stiffness, mass, and liquid viscosity of the damping device increased. Fu et al. $[34,35]$ devised a new passive variable damping energy dissipation device that the damping coefficient of it could be continuously changed with the external excitation speed and increased correspondingly when its speed increased. Wang et al. [36] developed the self-centering variable friction damper (SC-
VFD) brace and revealed that the SC-VFD brace had the same energy dissipation ability as the VFD brace, but the residual displacement was less and the equivalent viscous damping ratio was lower than that of the VFD brace.

The nonlinear damping isolator has a certain effect on the suppression of formant, the damping coefficient of the variable damping isolator can change with the change of external excitation, and its vibration isolation effect is better than that of the linear vibration isolator. In this paper, we proposed a nonlinear variable damping isolator with translating cam whose configuration is simpler than that before, and no additional energy input and complex control system are required. When the main system vibrates, the nonlinear variable damping isolator can provide a damping force, which varies with the change of its displacement, so that it can avoid infinitely large amplitudes at resonance and decrease the force transmissibility in the high-frequency region. The profile of the translating cam and the relative damping coefficient of the horizontal damper could be set up specially to meet the requirement of vibration isolation of the individual system.

The structure of this paper is as follows. The configuration of the proposed variable damping isolator is given, which comprises a translating cam and a pair of identical linear dampers arranged symmetrically and horizontally. Then static force analysis of the cam is conducted, and the dynamic equation of the proposed variable damping isolator is developed and solved by the harmonic equilibrium method. And afterward, their dynamic response under harmonic excitation is investigated. Finally, the force transmissibility of the variable damping isolator is given.

\section{Model of Nonlinear Variable Damping Isolator}

2.1. Configuration of Nonlinear Variable Damping Isolator. The nonlinear variable damping isolator with translating cam is shown in Figure 1. It is comprised of a translating cam and a pair of identical linear dampers arranged symmetrically and horizontally. The translating cam is provided with a curved track, and the damping cylinder of the linear damper is fixed. When the translating cam moves up and down, the roller drives the piston rod of the linear damper to move horizontally corresponding to the damping cylinder along the curved track so as to produce damping force.

2.2. Static Force Analysis of the Cam. To find the mechanism of the whole cam-roller, we may consider the mechanism of the right half side of the translating cam and roller on the basis of the principle of symmetry. The schematics of static force analysis of the right side of the translating cam and roller is illustrated in Figures 2(a) and 2(b), respectively, while external force $F$ in the $x$-axis direction acts on the variable damping isolator. In Figure 2(a), the external force $F$ includes the external excitation, the gravity of the cam, and the load. When the surface of the cam and roller is smooth, the friction force between them is so small that it might be negligible in comparison with the normal force $F_{n}$. If the cam 


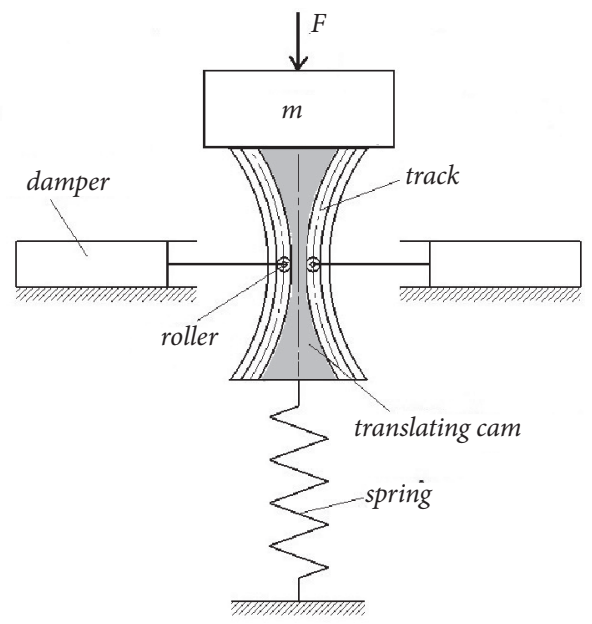

Figure 1: Schematic configuration of variable damping isolator.

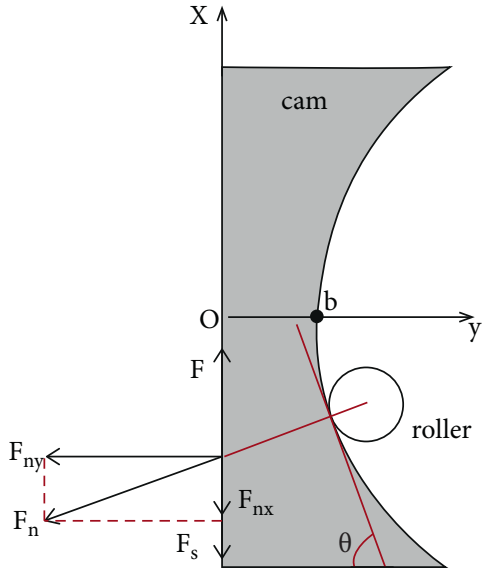

(a)

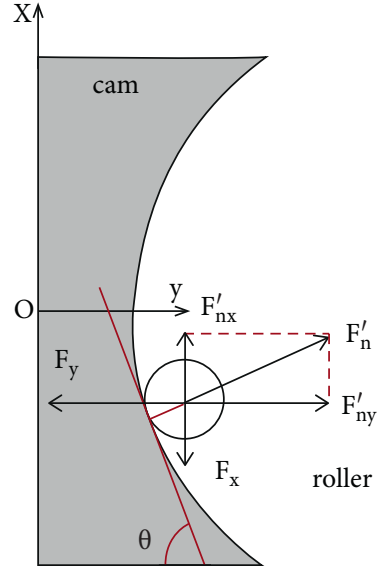

(b)

FIgURE 2: (a) The analysis schematics of the static force of the cam. (b) The schematics of the static force of the roller.

is force-balanced in the $x$-axis direction, its force equation is given as

$$
\left\{\begin{array}{l}
F=F_{s}+F_{n x}, \\
F_{s}=k_{s} x, \\
F_{n x}=F_{n} \cos \theta,
\end{array}\right.
$$

where $F_{S}$ is the elastic force of the vertical spring, $F_{n x}$ is the component of the normal force $F_{n}$ between the roller and cam in the $x$-axis, which may be defined as vertical damping force owing to the horizontal linear damper, $k_{s}$ is the stiffness of the spring, $\theta$ is the angle between the $y$-axis and tangent of the profile of the cam at the contact point of the cam and roller.

In Figure 2(b), when the roller is force-balanced in the $y$ axis direction, its force equation is as follows:

$$
F_{y}=F_{n}^{\prime} \sin \theta
$$

where $F_{n}^{\prime}$ is the reacting force of the normal force $F_{n}$ and $F_{n}^{\prime}$ is equal to $F_{n}$ and acts in a contrary direction. $F_{y}$ indicates the damping force of the horizontal linear damper.

Equation (3) is derived from equations (1) and (2).

$$
\tan \theta=\frac{F_{y}}{F_{n x}} .
$$

$\tan \theta$ may also be considered as the slope of the profile of the cam at the contact point. So equation (4) is given as follows:

$$
\tan \theta=\frac{\mathrm{d} x}{\mathrm{~d} y}
$$

Taking equation (3) into equation (4), we obtain $F_{n x}$ :

$$
F_{n x}=\frac{\mathrm{d} y}{\mathrm{~d} x} \cdot F_{y} .
$$


As the horizontal damper is a linear damper, $F_{y}$ is given as

$$
F_{y}=c^{\prime} \dot{y}
$$

where $c^{\prime}$ is the damping coefficient of the horizontal linear damper and $\dot{y}$ denotes a time derivative of horizontal displacement $y$.

Substituting equation (6) into equation (5) yields

$$
F_{n x}=\frac{\mathrm{d} y}{\mathrm{~d} x} \cdot c \dot{y} .
$$

As the damping force of the whole cam in the vertical direction is two times of $F_{n x}$, it is given as

$$
F_{\mathrm{d} x}=2 F_{n x}=2 c \dot{y} \cdot \frac{\mathrm{d} y}{\mathrm{~d} x},
$$

where $F_{\mathrm{d} x}$ is defined as the vertical damping force of the whole cam.

The vertical damping force may be expressed as the following form:

$$
F_{\mathrm{d} x}=c \dot{x}
$$

where $c$ is the damping coefficient of the vertical direction and $\dot{x}$ denotes a time derivative of vertical displacement $x$.

Based on equations (8) and (9), we can obtain the equivalent damping coefficient of the vertical direction $c$ as follows:

$$
c=2 c \prime\left(\frac{\mathrm{d} y}{\mathrm{~d} x}\right)^{2} .
$$

If the cam profile adopts a quadratic curve, we may write

$$
\mathrm{y}=\frac{a}{2} x^{2}+b
$$

where $a$ is the coefficient of the quadratic term of the quadratic curve and $b$ is one-half of the thickness of the cam in the $y$-axis direction as in Figure 2.

Based on equations (10) and (11), solving for $c$ results in

$$
c=2 c l(a x)^{2} \text {. }
$$

In equation (12), the vertical equivalent damping coefficient $c$ is directly proportional to the square of the vertical displacement $x$ of the variable damping isolator. Therefore, the variable damping isolator could provide variable damping force, while the vertical displacement of the system varies.

\section{Dynamic Performance of the Nonlinear Variable Damping Isolator}

3.1. Dynamic Equation. When the system moves up and down near the equilibrium position under harmonic excitation, the dynamic equation of the system may be formulated as

$$
m \ddot{x}+2 c(a x)^{2} \dot{x}+k x=n F_{0} \sin \omega t,
$$

where $m$ is the mass of the system, $x$ is the displacement of the system, the dot on it denotes a time derivative, $k$ is the stiffness of the spring, $n F_{0}$ is the amplitude of the excitation, and $\omega$ is the frequency of the excitation.

By making $L=F_{0} / k, T=\sqrt{m / k}, \bar{x}=x / L, \tau=t / T$, $\omega_{0}=\sqrt{k / m}, \Omega=\omega / \omega_{0}, \zeta=c / 2 \sqrt{k m}, b=a F_{0} / k$, (13) is nondimensionalized as

$$
\ddot{\bar{x}}+4 \zeta(b \bar{x})^{2} \dot{\bar{x}}+\bar{x}=n \sin \Omega \tau .
$$

To study the amplitude-frequency characteristics and force transmissibility of the variable damping system, the approximate analytical solution of the equation (14) is solved by the harmonic equilibrium method $[37,38]$.

Assume $\bar{x}=A \sin (\Omega \tau+\theta)$, and substitute it into equation (14). By neglecting the third-order term and setting the coefficients of the same harmonics equal, we can obtain equations (15) and (16).

$$
\begin{aligned}
\zeta b^{2} A^{3} \Omega & =n \cos \theta, \\
\Omega^{2} \mathrm{~A}-\mathrm{A} & =n \sin \theta .
\end{aligned}
$$

Both sides of equations (15) and (16) are squared, and the corresponding terms of them are added. Therefore, the system amplitude-frequency equation is obtained.

$$
\begin{aligned}
\zeta^{2} b^{4} \Omega^{2} A^{6}+\left(\Omega^{2}-1\right)^{2} A^{2} & =n^{2}, \\
\tan \theta & =\frac{\Omega^{2}-1}{\zeta b^{2} A^{2} \Omega} .
\end{aligned}
$$

According to equation (17), the amplitude $\mathrm{A}$ of the response is related to the horizontal damping ratio $\zeta$, the nondimensional property $b$, frequency ratio $\Omega$, and the nondimensional excitation amplitude $n$.

3.2. The Solution of Amplitude-Frequency Equation. Solving the amplitude-frequency equation by Shengjin's method is given as follows.

Substituting $x=A^{2}$ into equation(17), we can obtain

$$
\varsigma^{2} b^{4} \Omega^{2} x^{3}+\left(\Omega^{2}-1\right)^{2} x-n^{2}=0 .
$$

Thus, equation (19) has three roots, but only one real root as follows:

$$
\begin{aligned}
x= & -\frac{1}{3}\left[1.5\left(\frac{n^{2}}{\varsigma^{2} b^{4} \Omega^{2}}+\frac{\sqrt{81 n^{4} \varsigma^{2} b^{4} \Omega^{2}+12\left(\Omega^{2}-1\right)^{6}}}{\varsigma^{3} b^{6} \Omega^{3}}\right)\right]^{1 / 3} \\
& -\frac{1}{3}\left[1.5\left(\frac{n^{2}}{\varsigma^{2} b^{4} \Omega^{2}}-\frac{\sqrt{81 n^{4} \varsigma^{2} b^{4} \Omega^{2}+12\left(\Omega^{2}-1\right)^{6}}}{\varsigma^{3} b^{6} \Omega^{3}}\right)\right]^{1 / 3} .
\end{aligned}
$$

Thus, the amplitude A can be obtained as follows:

$$
A=\sqrt{x}
$$


3.3. Analytic Solution Validation. The direct numerical integral solutions of equation (14) by the fourth-order Runge-Kutta method are relied on to examine the validity of analytical approximate solutions. A diagram of the amplitude-frequency response is drawn as Figure 3, in which $\zeta=0.1, b=1$, and $n=1$. In Figure 3, the dynamic response curve of analytical approximate solutions is consistent with that of numerical solutions, while $\Omega>1.5$, but in the vicinity of low-frequency ratio region where the frequency ratio $\Omega$ is equal to 0.33 , the errors of analytical solution are about 10 percent. This is because the third-order terms $\zeta b^{2} A^{3} \Omega \cos 3(\Omega \tau+\theta)$ are omitted when the harmonic equilibrium method is adopted.

3.4. Frequency Response Analysis. Shengjin's method is adopted to solve equation (17). Then, nondimensional amplitude-frequency plots are drawn and analyzed.

When $n=1$ and $\zeta=0.1$, the nondimension amplitudefrequency is diagramed in Figure 4 , in which six curves represent $b=0.5,1,3,4,5$, and 7 , respectively. For $b=0.5$ and 1 , the amplitude has significant peaks in the vicinity of the frequency ratio $\Omega=1$. And the resonance amplitude while $b=0.5$ is larger than that while $b=1$. With the increase of $b$, the resonance amplitude decreases rapidly. When $b$ increases to 4 , the resonance peak disappears. This is because, in the resonance region, the sixth power of amplitude $A$ is reversely proportional to the fourth power of $b$ in the vicinity of the frequency ratio $\Omega=1$. In the region where the frequency ratio $\Omega \leq 2.5$, in the same frequency ratio, with the increase of $b$, the nondimension amplitude gradually decreases. In the region of frequency ratio $\Omega>2.5$, in the same frequency ratio, the different value of $b$ has little effect on the amplitude of response, and the amplitude of the response decreases with the increase of frequency ratio. Therefore, property $b$ could strongly affect the frequency of the response and resonance of the system. The greater its value (the greater the curvature of the profile of the cam) is, the larger the attenuation of the resonance of the response is.

When $n=1$ and $b=3$, the nondimensional amplitudefrequency diagram is given in Figure 5, in which five curves depict $\zeta=0.05,0.1,0.3,0.5$, and 0.7 , respectively. On the vicinity of the left side of the curves that the frequency ratio $\Omega=1$, when the relative damping coefficient $\zeta=0.05$ and 0.1 , the nondimension amplitudes have obvious peaks, and the smaller the nondimension resonance amplitude, the larger the relative damping coefficient $\zeta$. When the relative damping coefficient $\zeta=0.3$, the resonance phenomenon disappears. In the region where the frequency ratio $\Omega \leq 2.5$, in the same frequency ratio, with the increase of the relative damping coefficient, the amplitude gradually decreases. In the region where the frequency ratio $\Omega>2.5$, the change of the relative damping coefficient has little effect on the amplitude, and it decreases with the increase of the frequency ratio. The relative damping coefficient may also seriously affect the tendency of the frequency response curve and resonance peak of the response. The larger its value is, the higher the attenuation of the resonance of the response is.

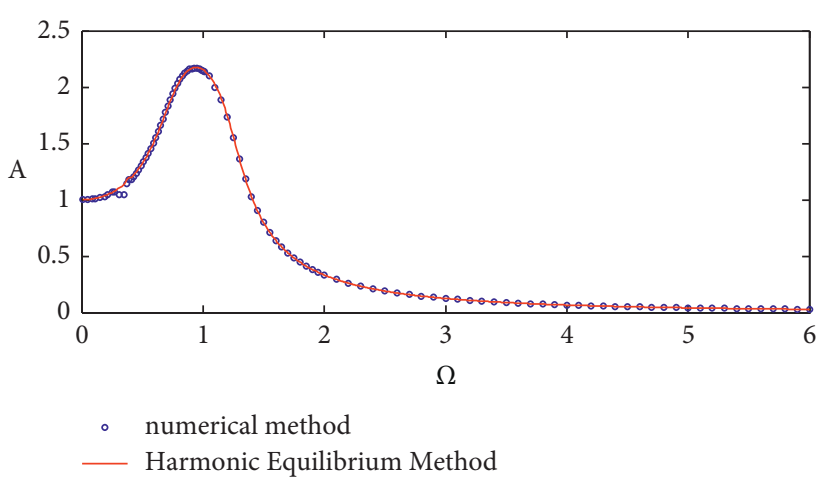

FIGURE 3: The response of nondimensional amplitude-frequency using harmonic equilibrium method and numerical method $(\zeta=0.1, n=1$, and $b=1)$.

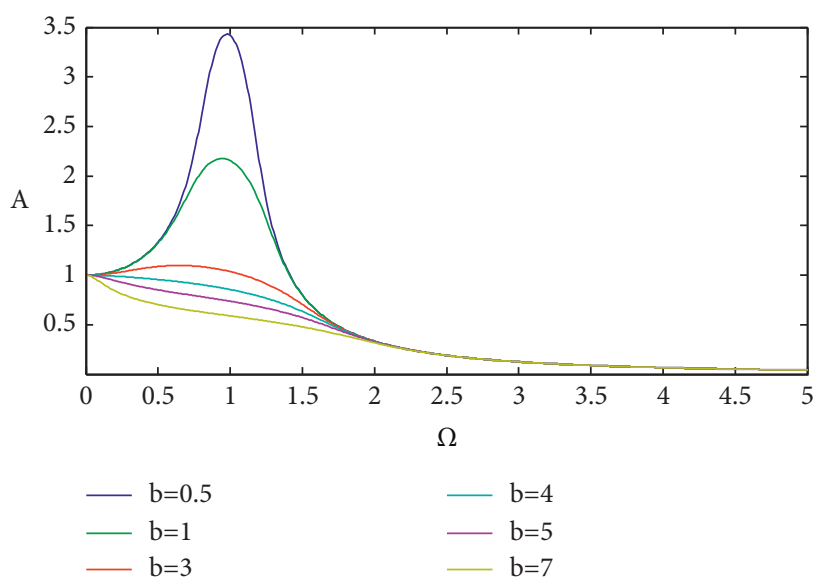

FIgURE 4: Amplitude-frequency ratio diagram for different $b$.

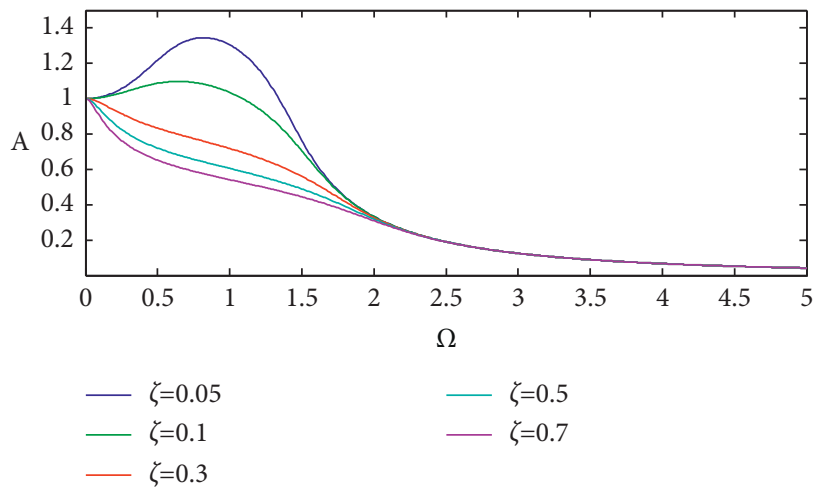

FIgURE 5: Amplitude-frequency ratio diagram for different relative damping coefficient $\zeta$.

In Figure 6, the nondimensional amplitude of the response for different frequency ratio diagram is illustrated when $\zeta=0.1$ and $b=3$. As we can see from Figure 6 , in the vicinity of the frequency ratio $\Omega=1$, when $n=0.1$ and 0.5 , the nondimension amplitudes have obvious resonance peaks, and with the increase of $n$, the resonance amplitude decreases obviously and shifts to the left gradually. When $n=2$ and 3 , there is no resonance phenomenon, and the 


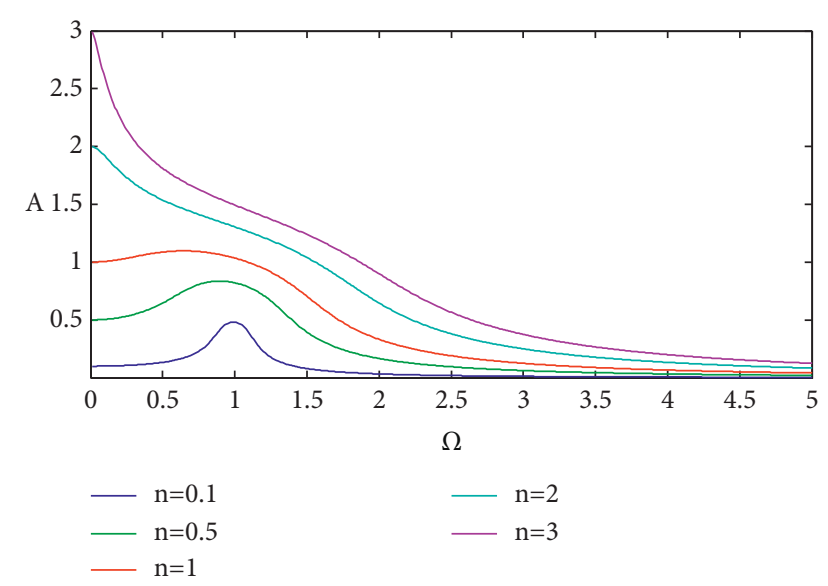

FIgURE 6: Amplitude-frequency ratio diagram for different $n$.

amplitudes attenuate gradually with the frequency ratio increasing. These show that the amplitudes of external excitations have an obvious effect on the amplitudes of responses. The larger the amplitude of the excitation is, the more strongly the resonant of the response is attenuated by the variable damping isolator.

3.5. Analysis of Force Transmissibility. Force transmissibility is an important index to evaluate vibration isolators. Force transmissibility is the ratio between the amplitude of the force transmitted to the foundation and the amplitude of the excitation. The force transmissibility [1] of the linear system is

$$
T_{0}=\sqrt{\frac{1+(2 \zeta \Omega)^{2}}{\left(1-\Omega^{2}\right)^{2}+(2 \zeta \Omega)^{2}}}
$$

where $T_{0}$ is the force transmissibility of the linear system.

The force transmitted to the foundation by the variable damping isolator is the sum of the damping force and spring force $[1,2]$ :

$$
\begin{aligned}
F_{T} & =4 \zeta(b x)^{2} \dot{x}+x \\
& =4 \zeta b^{2} A^{3} \Omega \sin ^{2} \Omega \tau \cos \Omega \tau+A \sin \Omega \tau \\
& =\zeta b^{2} A^{3} \Omega(\cos \Omega \tau-\cos 3 \Omega \tau)+A \sin \Omega \tau \\
& \approx \zeta b^{2} A^{3} \Omega \cos \Omega \tau+A \sin \Omega \tau \\
& =P_{T} \sin (\Omega \tau+\theta),
\end{aligned}
$$

where the third-order term $\cos 3(\omega \tau+\theta)$ is neglected. So the amplitude of the force transmitted to the foundation by the variable damping isolator is

$$
\begin{aligned}
P_{T} & =\sqrt{\zeta^{2} b^{4} A^{6} \Omega^{2}+A^{2}}, \\
\tan \theta & =\zeta b^{2} A^{2} \Omega .
\end{aligned}
$$

Therefore, the force transmissibility of the variable damping system is

$$
T_{1}=\frac{P_{T}}{P_{0}}=\frac{A}{n} \sqrt{\zeta^{2} b^{4} A^{4} \Omega^{2}+1},
$$

where $T_{1}$ is the force transmissibility of the variable damping system.

In equation (25), the force transmissibility is related to the excitation amplitude $n$, the damping ratio $\zeta$, and property $b$. Next, we shall illustrate our analysis of the effects of the parameters $n, b$, and $\zeta$ on the force transmissibility $T_{1}$.

When the damping ratio $\zeta=0.1$ and $b=3$, the force transmissibility of the variable damping system and the force transmissibility of the linear system are shown in Figure 7 , where five plots represent $n=0.1,0.5,1,3$, and 5 . As we can see from Figure 7 , the force transmissibility $T_{1}$ of the variable damping system and the linear system is all equal to 1 when $\Omega=\sqrt{2}$. In the region where $\Omega<\sqrt{2}$, the force transmissibility of the variable damping system is higher than that of the linear system when $n=0.1$, but the force transmissibility of the variable damping system is obviously lower than that of the linear system when $n=0.5,1,3$, and 5 . This shows that when the amplitude of excitation is small, the attenuating effect of the variable damping isolator on the formant is not as good as that of the linear isolator. As the excitation amplitude increases, the attenuating effect of variable damping isolator on formant will be better than that of linear vibration isolator. When the frequency ratio $\sqrt{2}<\Omega<2.35$ and $n=3$ and 5 , the force transmissibility of the variable damping isolator is a little greater than that of the linear isolator in the same frequency ratio. This shows that, in this region, with the increasing of the excitation amplitude, the attenuation of force transmissibility of variable damping isolator slows down, and the greater the excitation amplitude is, the greater the force transmissibility of variable damping isolator is. When $\Omega \geq 2.35$, the force transmissibility of the variable damping isolator is smaller than that of the linear system in the same frequency ratio. Therefore, the vibration isolation effect of the variable damping isolator is better than that of the linear system in most frequency ratios for big excitation amplitude.

When $\zeta=0.1$ and $n=1$, the force transmissibility of the variable damping isolator and the linear isolator in $b=0.5,1$, 3, 5, and 7 is illustrated in Figure 8. From Figure 8, it can be seen that the force transmissibility $T_{1}$ of the variable damping isolator and the linear isolator is equal to 1 when $\Omega=\sqrt{2}$. In the region where $\Omega<\sqrt{2}$, the force transmissibility of the variable damping system is greater than that of the linear system only when $b=0.5$, the force transmissibility of the variable damping system is clearly smaller than that of the linear system when $b=3,5$, and 7 , and the greater the $b$ gets, the better the suppression effect of the variable damping system on the resonance amplitude gets. However, when the value of $b$ increases to a certain extent, the inhibitory effect on the common amplitude value increases more and more slowly. This shows that the cam adopts a suitable curve, and the suppression effect of the variable damping vibration isolation system on the resonance amplitude is obviously better than that of the linear vibration isolation system, and the greater the curvature of the cam curve, the better the 


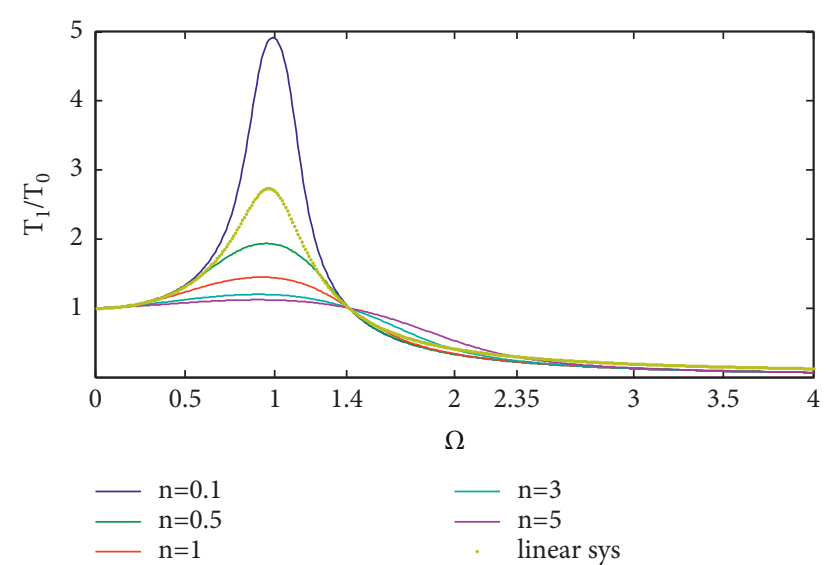

FIGURE 7: Force transmissibility-frequency ratio diagram for different $n$.

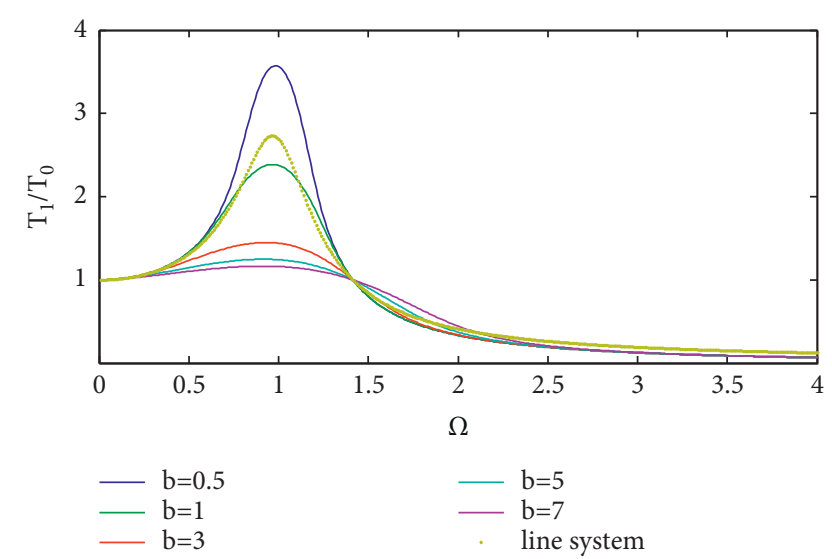

Figure 8: Force transmissibility-frequency ratio diagram for different $b$.

suppression effect on the resonance, but with the increase of curvature, the suppression effect on the common amplitude value increases more and more slowly. In the region of the frequency ratio $\sqrt{2}<\Omega<2.1, b=3$ and 5 , the force transmissibility of the variable damping system is slightly higher than that of the linear system. This means that, in this region, the larger the curvature of the cam of variable damping isolator is, the worse its vibration isolation effect becomes than that of the linear isolator. When the frequency ratio $\Omega \geq 2.35$, the force transmissibility of the variable damping system is smaller than that of the linear system. When the frequency ratio $\Omega>3$, with the increase of frequency ratio, the attenuation of force transmissibility of variable damping vibration isolation system slows down, and in the same frequency ratio, the force transmissibility in different $b$ values is almost the same. This shows that when designing the cam curve of variable damping vibrator, the attention may be focused on only the force transmissibility in the region where the frequency ratio is less than 3. And the greater the curvature of the cam curve is, the better the vibration isolation effect of the variable damper is almost.

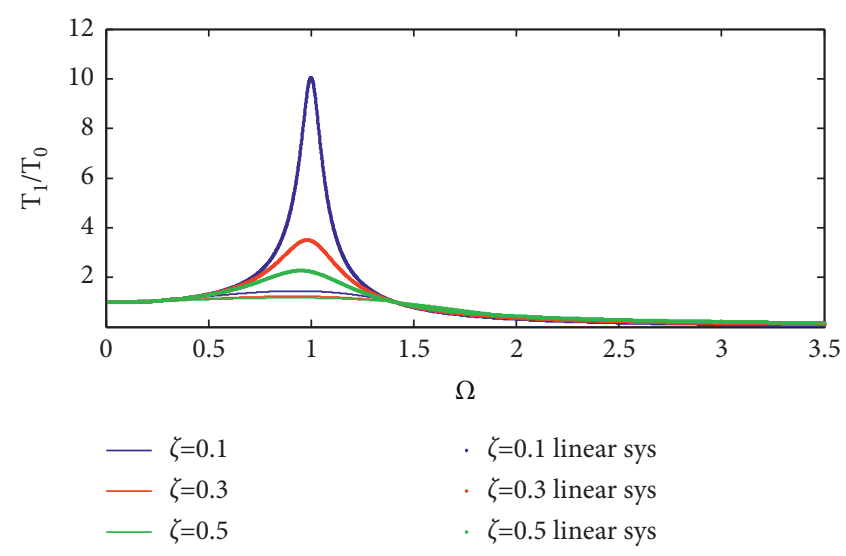

FIgURE 9: Force transmissibility-frequency ratio diagram for different relative damping coefficient.

When $b=3$ and $n=1$, the force transmissibility of the variable damping system and the linear system in relative damping coefficients that $\zeta=0.1,0.3$, and 0.5 is shown in Figure 9. It can be seen that the force transmissibility $T_{1}$ of the variable damping vibration isolation system and the linear vibration isolation system is equal to 1 when the frequency ratio $\Omega=\sqrt{2}$ in Figure 8 . In the region of the frequency ratio $\Omega<\sqrt{2}$, the force transmissibility of the variable damping vibration isolation system is obviously lower than that of the linear vibration isolation system in the same relative damping coefficient $\zeta$. However, the greater the relative damping coefficient $\zeta$ is, the slower the force transmissibility of the variable damping system decreases. When the frequency ratio $\sqrt{2}<\Omega<2$, the force transmissibility of the variable damping vibration isolation system is slightly higher than that of the linear vibration isolation system. And the greater the relative damping coefficient $\zeta$ is, the higher the force transmissibility of the variable damping system is. When $\Omega \geq 2$, the force transmissibility of variable damping vibration isolation system is lower than that of the linear system, with the frequency ratio increasing, the force transmissibility of variable damping system continues to reduce, and the force transmissibility of the variable damping system tends to be equal in different values of the relative damping coefficient. This shows that when selecting the relative damping coefficient of the horizontal damper of the variable damping system, we may just focus on the force transmissibility in the region of the frequency ratio $\Omega<2$, and it does not appear that with higher relative damping coefficient of the horizontal dampers comes the lower force transmissibility.

\section{Conclusion}

When the natural frequency of the system and the frequency of the excitation vary, to improve the isolation performance, the nonlinear variable damping isolator with translating cam is proposed. Its dynamic response and force transmissibility are derived through analytical and numerical methods.

(1) In the region of the low-frequency ratio, the nondimensional amplitude of the response decreases asymptotically with the increase of the property $b$ and 
the relative damping coefficient $\zeta$. In the region of the high-frequency ratio, the nondimensional amplitudes are almost equal in the same frequency ratio when the values of $b$ and $\zeta$ are different. The suppression of the resonance amplitude of the response is more obvious with the increase of $n, b$, and $\zeta$.

(2) When the amplitude of the external excitation is large, the suppression effect of the variable damping system on the formant is better than that of the linear vibration isolation system. In the region of the frequency ratio $\Omega<\sqrt{2}$, the larger the parameter $b$ is, the better the suppression effect on formants is. In the region of the high-frequency ratio, the vibration isolation performance of the variable damping vibration isolation system is better than that of the linear system.

Therefore, the variable damping system with translating cam is superior to the linear isolation vibration system in attenuating resonance in low-frequency region and isolating vibration performance in high-frequency region by setting appropriate cam curve and the damping coefficient of the horizontal damper. Next, we shall set up an experimental test system to verify the real isolation vibration performance of the variable damping isolator with translating cam.

\section{Data Availability}

The data used to support the findings of this study are included within the article.

\section{Conflicts of Interest}

The authors declare that they have no conflicts of interest.

\section{Acknowledgments}

The authors are grateful for the support by the National Natural Science Foundation of China (nos. U1934201 and 11772206).

\section{References}

[1] Z. C. Zheng, Mechanical Vibration (First Volume), Machinery Industry Press, Beijing, China, 1980.

[2] P. D. Dai, Damping Technology for Vibration and Noise Control, Xi'an Jiaotong University Press, Xi'an, China, 1986.

[3] E. I. Rivin, Passive Vibration Isolation, ASME Press, New York, NY, USA, 2001.

[4] B. Ravindra and A. K. Mallik, "Hard Duffing-type vibration isolator with combined Coulomb and viscous damping," International Journal of Non-linear Mechanics, vol. 28, no. 4, pp. 427-440, 1993.

[5] B. Ravindra and A. K. Mallik, "Performance of non-linear vibration isolators under harmonic excitation," Journal of Sound and Vibration, vol. 170, no. 3, pp. 325-337, 1994.

[6] R. A. Lbrahim, "Recent advances in nonlinear passive vibration isolators," Journal of Sound and Vibration, vol. 314, pp. 371-452, 2008.

[7] J. Teng, Z. X. Lu, Y. Q. Xiao, A. Z. Yan, and C. T. Li, "Vibration control of high-rising buildings with TMD considering contract non-linear damping effect," Journal of Vibration and Shock, vol. 28, pp. 90-96, 2009.

[8] H. He, P. Tan, Y. H. Liu, and Y. Xiang, "Wind-induced vibration control of circular section high-rise structures employing TMD with two-stage damping level," Journal of Vibration and Shock, vol. 33, pp. 503-508, 2020.

[9] Y. Zhang and S. Zhu, "A shape memory alloy-based reusable hysteretic damper for seismic hazard mitigation," Smart Materials and Structures, vol. 16, no. 5, pp. 1603-1613, 2007.

[10] Y. Zhang and S. Zhu, "Seismic response control of building structures with superelastic shape memory alloy wire dampers," Journal of Engineering Mechanics, vol. 134, no. 3, pp. 240-251, 2008.

[11] S. M. Gong and Y. Zhou, "Experimental study and numerical simulation on a new type of viscoelastic damper with strong nonlinear characteristics," Struct. Control Hlth.vol. 24, pp. 1-18, Article ID e1897, 2007.

[12] Z. Huang, H. N. Li, and X. Fu, "Optimum design of a recentering deformation-amplified SMA damper," Engineering Mechanics, vol. 36, pp. 202-210, 2019.

[13] H.-N. Li, Z. Huang, X. Fu, and G. Li, "A re-centering deformation-amplified shape memory alloy damper for mitigating seismic response of building structures," Structural Control and Health Monitoring, vol. 25, no. 9, pp. 1-20, Article ID e2233, 2018.

[14] H. N. Li, Z. Huang, and X. Fu, "Study on the performance of a gear-driven rotation-amplified rubber viscoelastic damper and its vibration control for structures," Struct. Control Hlth., vol. 27, Article ID e2617, 2020.

[15] J. Rabinow, "The magnetic fluid clutch," Transactions of the American Institute of Electrical Engineers, vol. 67, no. 2, pp. 1308-1315, 1948.

[16] P. P. Pradeep, "Magnetorheological(MR) fluids: principles and applications," Smart Materials Bulletin, vol. 2001, pp. 7-10, 2001.

[17] S. Dong, K.-Q. Lu, J. Q. Sun, and K. Rudolph, “A prototype rehabilitation device with variable resistance and joint motion control," Medical Engineering \& Physics, vol. 28, no. 4, pp. 348-355, 2006.

[18] Y. Zhou and P. Tan, Theory and Technology of Magnetorheological Damping Control, Science Press, Beijing, China, 2007.

[19] W. L. Wang, D. S. Yu, Y. Huang, Z. Zhou, and R. Xu, “A locomotive's dynamic response to in-service parameter variations of its hydraulic yaw damper," Nonlinear Dynamics, vol. 77, no. 4, pp. 1485-1502, 2014.

[20] S. M. Mousavi-Bideleh and V. Berbyuk, "Multiobjective optimization of bogie suspension to boost speed on curves," Vehicle System Dynamics, vol. 54, pp. 1-27, 2016.

[21] S. Wang, Y. Liu, D. Li, and X. Z. He, "A ferrofluid-based planar damper with magnetic spring," Journal of Magnetics, vol. 23, no. 3, pp. 405-408, 2018.

[22] X. Jiao, Y. Zhao, and W. Ma, "Nonlinear dynamic characteristics of a micro-vibration fluid viscous damper," Nonlinear Dynamics, vol. 92, no. 3, pp. 1167-1184, 2018.

[23] T. H. Jin, Z. M. Liu, Z. S. Ren, X. J. Zhang, and X. Li, "Adaptability of variable stiffness and damping shock absorber for semi-active suspension of high speed train," Journal of Vibration Engineering, vol. 33, pp. 772-783, 2020.

[24] J. Yu, X. Dong, S. Qi, T. Wang, and Y. Liang, "Development of a magnetorheological isolator with variable damping and variable stiffness for broadband vibration suppression," Smart Materials and Structures, vol. 30, no. 2, Article ID 025023, 2021. 
[25] Z. K. Peng, Z. Q. Lang, L. Zhao, S. A. Billings, G. R. Tomlinson, and P. F. Guo, "The force transmissibility of MDOF structures with a non-linear viscous damping device," International Journal of Non-linear Mechanics, vol. 46, no. 10, pp. 1305-1314, 2011.

[26] Z. K. Peng, G. Meng, Z. Q. Lang, W. M. Zhang, and F. L. Chu, "Study of the effects of cubic nonlinear damping on vibration isolations using Harmonic Balance Method," International Journal of Non-linear Mechanics, vol. 47, no. 10, pp. 10731080, 2012.

[27] X. T. Liu, S. H. Chen, J. D. Wang, and J. F. Shen, "Analysis of the dynamic behavior and performance of a vibration isolation system with geometric nonlinear friction damping," Chinese Journal of Theoretical and Applied Mechanics, vol. 51, pp. 371-379, 2019.

[28] V. T. Vu, O. Sename, L. Dugard, and P. Gaspar, "Active antiroll bar control using electronic servo valve hydraulic damper on single unit heavy vehicle," IFAC-PapersOnLine, vol. 49, no. 11, pp. 418-425, 2016.

[29] D. D. Domenico and G. Ricciardi, "Earthquake protection of structures with nonlinear viscous dampers optimized through an energy-based stochastic approach," Engineering Structures, vol. 179, pp. 523-539, 2019.

[30] L. Wang, P. Zhou, and M. L. Xia, "Structure optimization and simulation based on AMESim for hydraulic damper," Chinese Hydraulics \& Pneumatics, vol. 6, pp. 94-98, 2018.

[31] M. C. Yu, B. W. Li, Z. L. Niu, W. K. Xue, and P. Zhao, "Dynamical properties of variable-damped vibration isolator based on shear thickening fluid," Equipment Environmental Engineering, vol. 16, pp. 33-38, 2019.

[32] M. Yan, H. N. Tian, and H. C. Liu, "Nonlinear damping characteristics of pore-type vibration isolation system," Journal of Shenyang University of Technology, vol. 42, pp. 648-653, 2020.

[33] X. Zhao, Y. M. Gao, T. G. Zhou, W. Q. Li, and Y. J. Ding, "Mechanical performance test and parameter ortimization of a new variable damping TMD," Journal of Xi'an University of Architecture and Technology, vol. 52, pp. 797-805, 2020.

[34] W. Q. Fu, M. Li, and C. W. Zhang, "Design and performance experiment on passive variable damping energy dissipation device," Journal of Vibration Engineering, vol. 33, pp. 869876, 2020.

[35] W. Q. Fu, M. Li, T. Li, and C. W. Zhang, "Design, performance test and structural wind vibration control analysis of multi-stage variable damping device," Engineering Mechanics, vol. 37, pp. 225-233, 2020.

[36] Y. Wang, Z. Zhou, Q. Xie, and L. Huang, "Theoretical analysis and experimental investigation of hysteretic performance of self-centering variable friction damper braces," Engineering Structures, vol. 217, Article ID 110779, 2020.

[37] A. H. Nayfeh and D. T. Mook, Nonlinear Oscillations, John Wiley \& Sons, New York, NY, USA, 1979.

[38] Y. S. Chen, Nonlinear Vibration, Higher Education Press, Beijing, China, 2002. 